\title{
Evaluation of Effects and Mechanism of Hedysarum Polybotys Saccharide on Ventricular Remodeling in Mice Model
}

\author{
Liu Bo ${ }^{1}$, Weisi Feng ${ }^{1}$, Qiuyang $\mathrm{Yu}^{1}$, Mingcheng $\mathrm{Li}^{1 \text {, a }}$, Ruowen Zhang ${ }^{2, \mathrm{~b}}$ \\ ${ }^{1}$ Department of Pathogenic Biology, School of Medicine laboratory, Beihua University, Jilin 132013, \\ China \\ ${ }^{2}$ Department of Medicine, Beihua University, Jilin 132013, China \\ 1, a Correspondence author: e-mail: limingcheng@beihua.edu.cn; ${ }^{2, b}$ e-mail:zrw1828@163.com
}

Keywords: Hedysarum Polybotys Saccharide; isoprenaline; ventricular remodeling

\begin{abstract}
Objective To study the effects and mechanism of Hedysarum Polybotys Saccharide (HPS) on ventricular remodeling induced by isoprenaline (ISO) in the mouse. Methods Ventricular remodeling model was induced by subcutaneous injections of ISO for continuous fourteen days. Mice were randomly divided into control group, ISO group, low-dose group, middle-dose group and high-dose group of HPS were introduced by intergalactic (fig.) administration for twenty one days.Cardiac mass index of mouse was calculated at the end of experiment, hydroxyproline (Hyp) content, nitric oxide (NO) content, nitric oxide synthase (NOS) activity, superoxide dismutase (SOD) activity and malondialdehyde (MDA) content in myocardial tissue were measured by spectrophotometry. Results Compared with control group, the heart weight index and Hyp content in ISO group was significantly increased $(p<0.01)$, the SOD activity was decreased $(p<0.01)$ and MDA content was increased $(p<0.01)$, the NOS activity and NO level were reduced $(p<0.05$, $p<0.01)$. Compared with the ISO group, HPS group could reduce the heart weight index obviously $(p<0.05)$, inhibited the Hyp content, increased SOD activity and reduced MDA content, enhanced NOS activity and NO level in myocardium $(p<0.05$ and $p<0.01)$. Conclusion HPS have inhibitory effects on ventricular remodeling induced by ISO in mice. Its mechanisms may be related to increasing NOS activity and NO level, in addition to enhancing the antioxidant ability of heart.
\end{abstract}

\section{Introduction}

Ventricular remodeling (VR) is referred to the changes of ventricular structure, including cardiac myocyte hypertrophy and interstitial cells, such as fibroblast (CFB) proliferation and collagen tissue hyperplasia. Ventricular remodeling is often secondary to hypertension, myocardial infarction and ischemic cardiomyopathy, arrhythmia, heart failure, and sudden death occurred gradually with the progress of the disease, therefore, the early use of drug intervention to reverse cardiac hypertrophy and prevent ventricular remodeling are effective means for the treatment of congestive heart failure.

Astragalus and Radix Hedysari belong to the same family, but different genera. Both are the specialty of Gansu. Radix Hedysari polysaccharide is the main active component of Radix Hedysari and its content is about twenty to thirty four percent. It has the function of benefiting Qi [1] [2] enriching blood, diuretic, detoxification, regulating immunity, antitumor etc. In recent years, it was reported that Radix Hedysari polysaccharides has the effects of reducing glucose,modulating lipid,improving insulin resistance and resisting myocardial hypertrophy induced by diabetes [3]. It has a good potential in the treatment of diabetes mellitus. This experiment is aimed to explore the effects of Radix Hedysari polysaccharide on ventricular remodeling induced by isoproterenol and its preliminary mechanism in order to provide a theoretical basis. For revealing the protective effects on heart and probing the efficacy ingredients further.

\section{Materials and methods}

Experimental animals. Clean and healthy ICR mice, with no limit for male and female, body weight between $(20 \pm 2)$ gram, were provided by the experimental animal research center of Jilin 
University, experimental animal production license No. SCXK (Ji): 2011-0007. With cage feeding, adequate feed, drinking water is not limited, room temperature is about twenty to twenty five degrees centigrade. All mice were applied after adaptation to the environment for five days.

Experimental drugs and reagents. The extraction and separation of polysaccharides: Take one hundred grams of prepared Radix Hedysari granule precisely and by ethanol degreasing, adding eight times amount of distilled water decoction, concentrating the filtrate respectively by the concentration of sixty and eighty percent of ethanol cascade precipitation, sediment by savage method repeatedly to protein, vacuum drying, refining Radix Hedysari Polysaccharide (HPS). Radix Hedysari Polysaccharide content determination: Accurately weigh 1.000 g of dried skim Hedysarum Polybotrys hand, add distilled water decoction, constant volume to $100 \mathrm{ml}$, headspace sample solution $2.0 \mathrm{ml}$ by phenol sulfuric acid method determination of absorbance and by the linear regression equation calculation sample solution of Radix Hedysari Polysaccharide content according to the material liquid ratio $1: 8(\mathrm{w} / \mathrm{ml}$, the followings are the same). Isoproterenol was purchased from Shanghai Hefeng pharmaceutical company, batch number for 20120203; enzyme and nitric oxide ELISA kit for superoxide discrimination enzyme reagent box, malondialdehyde kit, hydroxyproline kit, nitric oxide synthase were provided by Nanjing Jiancheng engineering research, the batch No. 20120824.

Animal grouping and treatment. The pure lines of Kunming mice, with half males and half females, were randomly divided into five groups, fifteen rats in each group. Blank control group (10 rats), model group(ISO,15 rats), low dose group of Hedysarum Polybotys Saccharide (HPS $100 \mathrm{mg} / \mathrm{kg}, 15$ rats), medium dose group (HPS 200mg/kg, 15 rats), high dose group (HPS 400mg/kg, 15 rats). During the experiment, there were dead animals in each group by accident, so the final samples are different. Ventricular remodeling model in mice was made according to the literature [4]. Control group was injected with corresponding capacity of saline. At the same time, the drug was administered once per day, for consecutive twenty-one days, and the volume of gastric administration was $0.02 \mathrm{ml} / \mathrm{g}$ body weight. Model group and blank control group were used with equal volume of distilled water. After the final administration, fasting 24h, all animals were killed in the twenty-two days.

Cardiac weight index. Weighing the body weight, the mice were sacrificed by cervical dislocation after taking blood of orbital vein, opening the chest quickly, taking the heart out, washing the residual blood with normal saline and drying with filter paper. Weighing the heart mass by an electronic balance, calculating the heart weight/body weight ratio (HW/ BW, mg/g) and left ventricular weight/weight ratio ( $\mathrm{LVM} / \mathrm{HW}, \mathrm{mg} / \mathrm{mg})$.

Determination of SOD activity and MDA content in myocardium. Taking about $100 \mathrm{mg}$ of myocardial tissue, tissue homogenate was prepared by $5 \%$ saline in the ice bath, 4 degrees centigrade, $4000 \mathrm{r} / \mathrm{min}$ centrifugation for $15 \mathrm{~min}$, the supernatant measured strictly in accordance with the kit instruction. The activity of SOD was determined by xanthine oxidase method and the content of MDA was measured by phenobarbitone acid method.

Determination of hydroxyproline content in myocardial tissue. 50mg of apical myocardial tissues was washed with saline, cut into pieces, mixed with $1 \mathrm{ml}$ of hydrolysate and then put into the test tube of glass ground joint with cover, stamped, bathed under ninty-five degrees centigrade or boiling water for hydrolysis of $20 \mathrm{~min}$, adding reagent in accordance with the instructions sequentially, $3500 \mathrm{r} / \mathrm{min}$ centrifugation for $10 \mathrm{~min}, 1 \mathrm{ml}$ supernatant was taken for detection, determining the hydroxyproline content according to the Nanjing Jiancheng Bioengineering Institute of medicine and instructions, the resulting value $/ 0.134$ is the content of collagen in myocardial tissue.

Determination of NOS activity and NO level in myocardium. NO was determined by nitrate reductase method, detecting NO level in cell culture fluid, read the absorbance of color depth. NO content in the samples is according to the formula [(determination tube absorbance of the blank tube absorbance) / (standard tube absorbance of the blank absorbance tube) $100 \mu \mathrm{mol} / \mathrm{l}$ ] calculation. NOS activity was measured by a spectrophotometric method. NOS activity in the amount of NO was 
generated per minute, according to the formula [(determination tube absorbance of the blank absorbance tube) $141.6 \mathrm{U} / \mathrm{ml}$ ] calculation.

Statistical methods. Using SPSS 10 statistical analysis software for statistical analysis. All data were expressed in $(x \pm s)$, single factor analysis of variance was used between groups comparison and LSD-t test was compared between the two groups, $p<0.05$ meaning that the differences have statistical significance.

\section{Results}

Effects of HPS on ventricular weight/body weight(LVM/BW), heart weight index (LVI) and hydroxyproline (Hyp) content in ISO-induced ventricular remodeling mouse. Mice for subcutaneous injections of ISO for 14 days later, compared with the control group, HW/BW, LVI and the content of hydroxyproline in model group increased significantly $(p<0.05$ or $p<0.01)$. Low, middle and high dose of HPS groups inhibited the increase of HW/BW, LVI and reduced the content of hydroxyproline, the difference is statistically significant compared with the model group, $(p<0.05, p<0.01)$, indicating that HPS can inhibit ventricular remodeling induced by ISO. Results were shown in table 1.

Effects of HSP on NOS, SOD activity and MDA, NO level in mice myocardial tissue of ISOinduced ventricular remodeling. It was showed that, compared with the control group. NOS activity and NO level in ISO group decreased significantly $(p<0.05$ or $p<0.01)$ in Table 2. SOD activity significantly decreased, but MDA content increased in ISO group, compared with those in control group $(p<0.01)$, HPS of each doses of groups could increase NOS, SOD activity and NO level, decreased MDA content, which have very significant differences compared with those of ISO group ( $p<0.05, p<0.01$ or $p<0.001)$.

Tab.1 Effects of HPS in HW/BW,LVI, Hyp content $\left(n=15,{ }^{X} \pm s\right)$

\begin{tabular}{|c|c|c|c|c|}
\hline Group & $\begin{array}{c}\text { Dose } \\
\left(\mathrm{mg}^{\left.-\mathrm{kg}^{-1}\right)}\right.\end{array}$ & $\begin{array}{c}\mathrm{HW} / \mathrm{BW} \\
\left(\mathrm{mg} \cdot \mathrm{kg}^{-1}\right)\end{array}$ & $\begin{array}{c}\mathrm{LVW} / \mathrm{HW}(\mathrm{LVI}) \\
\left(\mathrm{mg} \cdot \mathrm{kg}^{-1}\right)\end{array}$ & $\begin{array}{c}\mathrm{Hyp} \\
\left(\mu \mathrm{g} \cdot \mathrm{kg}^{-1}\right)\end{array}$ \\
\hline Control & - & $3.489 \pm 0.362$ & $0.325 \pm 0.036$ & $0.21 \pm 0.04$ \\
\hline ISO & - & $6.056 \pm 1.82^{*}$ & $0.752 \pm 0.054^{* *}$ & $0.58 \pm 0.08^{* *}$ \\
\hline SCL-L & 100 & $4.596 \pm 0.378^{\Delta}$ & $0.570 \pm 0.025^{\Delta}$ & $0.33 \pm 0.05^{\Delta}$ \\
\hline SCL-M & 200 & $3.510 \pm 0.321^{\Delta \Delta}$ & $0.494 \pm 0.065^{\Delta}$ & $0.30 \pm 0.03 \Delta \Delta$ \\
\hline SCL-H & 400 & $3.577 \pm 0.362^{\Delta \Delta}$ & $0.471 \pm 0.0657 \Delta \Delta$ & $0.27 \pm 0.04 \Delta \Delta$ \\
\hline
\end{tabular}

$* p<0.05$, $* * p<0.01$, VS Control group; $\Delta p<0.05, p<0.01$, VS ISO group

Tab.2 Effects of HPS in NOS, SOD activity and MDA, NO levels ( $\left.\mathrm{n}=15, \bar{X}_{ \pm \mathrm{s}}\right)$

\begin{tabular}{|c|c|c|c|c|c|}
\hline Group & $\begin{array}{c}\text { Dose } \\
(\mathrm{mg} \cdot \mathrm{kg}-1)\end{array}$ & $\begin{array}{c}\mathrm{NOS} \\
{[\lambda \mathrm{B}(\mathrm{U} \cdot \mathrm{ml}-1)]}\end{array}$ & $\begin{array}{c}\text { NO } \\
{[\mathrm{cB} /(\mu \mathrm{mol} \cdot \mathrm{L}-1)]}\end{array}$ & $\begin{array}{c}\text { SOD } \\
{[\lambda \mathrm{B} /(\mathrm{U} \cdot \mathrm{mg}-1 \cdot \mathrm{prot})}\end{array}$ & $\begin{array}{c}\text { MDA } \\
[\mathrm{mB} / \mathrm{nmol} \cdot \mathrm{mg}-1 \mathrm{prot})]\end{array}$ \\
\hline Control & - & $60.54 \pm 9.53$ & $30.45 \pm 4.21$ & $30.65 \pm 4.12$ & $1.67 \pm 0.45$ \\
\hline ISO & - & $38.60 \pm 7.14^{* *}$ & $20.32 \pm 3.50^{*}$ & $16.22 \pm 2.45^{* *}$ & $5.16 \pm 0.47^{* *}$ \\
\hline SCL-L & 100 & $45.13 \pm 7.26^{\Delta}$ & $26.78 \pm 3.04^{\Delta}$ & $24.78 \pm 3.15^{\Delta}$ & $3.75 \pm 0.17$ \\
\hline SCL-M & 200 & $50.74 \pm 8.25^{\Delta \Delta}$ & $35.19 \pm 4.62^{\Delta \Delta}$ & $28.87 \pm 3.16^{\Delta}$ & $2.17 \pm 0.43^{\Delta \Delta}$ \\
\hline SCL-H & 400 & $58.27 \pm 7.16^{\Delta \Delta}$ & $38.76 \pm 3.80^{\Delta \Delta}$ & $35.70 \pm 3.15^{\Delta \Delta \Delta}$ & $2.01 \pm 0.87 \Delta \Delta$ \\
\hline
\end{tabular}

${ }^{*} p<0.05$, ** $p<0.01$, VS Control group; ${ }^{*} p<0.05, p<0.01, p<0.001$, VS ISO group 


\section{Discussion}

Ventricular remodeling is caused by a series of complex molecular and cellular mechanisms of myocardial structure, function and phenotypic changes, mainly manifested as myocardial function change, myocardial hypertrophy and myocardial fibrosis. Continuous subcutaneous injections of ISO induced VR is classical in animal models of cardiac hypertrophy, ISO can promote increased myocardial cell total protein and contractile protein synthesis, myocardial hypertrophy and interstitial fibrosis, resulting in VR eventually, characterized by increased heart weight and extracellular matrix collagen fiber mass deposition [5]. ISO is a beta receptor agonist and its positive inotropic and chronotropic effects enhanced the myocardial contractility, accelerated heart rate, hypertrophic myocardial myocyte and hyperplasia of collagen fibers, resulting in myocardial hypertrophy [6].

Radix Hedysari is dry roots of legume sequence of Hedysaryum Polybotrys Hand Mazz, it is called HongQi because of the red color of skin, it is referred to as the Radix Hedysari. The original plant and the HuangQi belong to the same family of different genera, since ancient times Radix Hedysari and Astragalus shared general purpose. Radix Hedysari has effects of invigorating qi and consolidation of superficies, diuretic and detoxification,nourishing blood, discharging pus, promoting tissue regeneration and sore healing function, commonly used in deficiency of vital energy, fatigue, sinking of Qi, less food, Hyperhidrosis, diarrhea frequently, rectocele, heat diabetes and other diseases treatment [7]. Past research on Radix Hedysari are more concentrated on immune regulation, in recent years, the study found that Radix Hedysari also has hypoglycemic, antihypertensive, modulating blood lipid and improving insulin resistance [8][9], it has good curative effect in the treatment of hypertension, diabetes, ischemic cardiomyopathy, arrhythmia, cerebral vascular accident, cerebral infarction and chronic kidney disease. Radix Hedysari polysaccharide (HPS) is the main active ingredient of Radix Hedysari.

Polysaccharides of different molecular weight were produced by alcohol precipitation in Madan's research of HongQi. Determination of polysaccharide structure with different molecular weight in chromatography found that different polysaccharide are all constituted by monosaccharide of glucose, rhamnose, galactose, xylose, arabinose, etc. in different ratios [10]. The study concluded that extract of Radix Hedysari by water saturated and n-butanol has scavenging effects on superoxide anion radical [11].

NO holds the function of regulating blood vessel tension, antioxidation, anti-inflammation and inhibiting the proliferation of vascular smooth muscle. Recent studies have found that NO has a definite role in the regulation of myocardial fibrosis and collagen deposition [12]. Research showed that continuous subcutaneous injections of ISO could significantly increase oxidative stress and lead to the occurrence of VR. SOD is an important antioxidant enzyme in myocardium, which plays an important role in the process of protecting myocardial cells from oxidative damage. MDA is the major end products of lipid peroxidation. So the measurement of myocardial SOD activity and MDA content can reflect the level of oxygen free radicals and lipid peroxidation degree of organism [13]. In recent years, a large number of studies revealed that there is a close relationship between oxidative stress and VR. Oxygen free radicals induced myocardial cell hypertrophy and apoptosis by direct or indirect signal transduction pathway, resulting in VR.

The experimental results showed that HPS can significantly reduce the HW/BW and LVM/HW ratio in VR induced by ISO, reduced the content of hydroxyproline, increased the activity of NOS, NO levels and increased SOD activity, decreased MDA level compared with that of model group ( $p<0.05, p<0.01$ or $p<0.001$ ), indicating that HPS has obvious inhibitory effects on VR induced by ISO.

In summary, HPS inhibited the occurrence of VR, the primary mechanism is to increase the NOS activity, thereby increasing NO synthesis, increase the activity of SOD, decrease MDA level in myocardial tissue, reduce myocardial collagen deposition, improve the body's antioxidant capacity. 


\section{Acknowledgement}

This project was financially supported by Health Developing Program in Jilin Province, China (Contract No. 20132099) and Science and Technology Research Projects of Department of Education of Jilin Province, China (Contract No. 2011-132).

\section{References}

[1] Liu Zhong. Experimental study of Hedysarum Polybotys Saccharide extraction and anti-tumor effect[D] master, Lanzhou University, 2007.

[2] Liu Hua, Li Yi, Li Ping-yan, et al. Radix Hedysari polysaccharide anti-tumor and immune regulation effect [J]. International Journal of oncology, J Clin Oncol, 2011, 38 (8): 589-591.

[3] Zhi Sheng-jin, Li Juan-e, Zhang Dong-peng et al. Effects of Radix Hedysari polysaccharide to improve type 2 diabetic rat model of insulin resistance effect [J]. Chinese Journal of medicine, 2007, 14 (5): $27-28$.

[4] Zhao W, Zhao D, Yan R, et al. Cardiac oxidative stress and remodeling following infarction: role of NADPH oxidase [J]. Cardiovasc Pathol, 2009, 18: 156-166.

[5] Fan GC, Yuan Q, Song G, et al. Small heat-shock protein Hsp20 attenuates beta-agonist-mediated cardiac remodeling through apoptosis signal-regulating kinase 1[J]. Circ Res, 2006, 99: 1233-1242.

[6] Bos R, Mougenot N, Findji L, et al. Inhibition of cate-cholamine - induced cardiac fibrosis by an aldosterone antagonist[J]. Cardiovasc Pharmacol, 2005,45(1):8-13.

[7] Huang Zhao-sheng. Chinese pharmacy, Beijing people's Medical Publishing press, 2002,49.

[8] Liang Yi-qiang, Pan Zhao-xin, He Xin-bing. Research progress in the pathogenesis of ventricular remodeling [J]. Hebei medicine, 2013, 35 (24): 3781-3784.

[9] Lin Ze-peng, Zhang Zhi-wei, Zhao You-sheng et al. Irbesartan in long-term treatment of primary hypertension patients with left ventricular hypertrophy and cardiac function ". Chinese Journal of clinical pharmacology and therapeutics, 2005, 10 (2): 219-221.

[10] Liu Qin-deng, Wu Bao-yi, Chen Jie, Du Jie-ying. Traditional Chinese medicine Radix Hedysari,chemical components and pharmacological effects [J]. Pharmaceutical economics in China, 2013, 9:36-39.

[11] Liu Kai, Xu Zhong, Ying Dong-li. Radix Hedysari polysaccharide promote the angiogenesis of chick chorioallantoic membrane (CAM) experimental study [J]. TCM research, 2008, 21(12):14-16.

[12] Du Jun, Chen chang-xun. Chinese intervention in ventricular remodeling on nitric oxide pathway research progress [J]. Journal of integrated traditional Chinese and Western medicine, 2007, 5 (6): 702-706.

[13] Rajadurai M, Stanely Mainzen Prince P. Preventive effect of naringin on lipid peroxides and antioxidants in isoproterenol-induced cardiotoxicity in Wistar rats: biochemical and histopathological evidences[J]. Toxicology, 2006, 228: 259-268. 